\title{
BMJ Open Intake of marine $n-3$ polyunsaturated fatty acids and the risk of rheumatoid arthritis: protocol for a cohort study using data from the Danish Diet, Cancer and Health cohort and Danish health registers
}

Bolette Gylden Soussi (10 ,' Christian Sørensen Bork (1) ,2 Salome Kristensen,, ${ }^{1,3}$ Søren Lundbye-Christensen, ${ }^{4,5}$ Kirsten Duch, ${ }^{4}$ René Lindholm Cordtz, ${ }^{1}$ Jeppe Hagstrup Christensen, ${ }^{3,6}$ Erik Berg Schmidt, ${ }^{2,3}$ Lene Dreyer $^{1,3}$

To cite: Soussi BG, Bork CS, Kristensen S, et al. Intake of marine $\mathrm{n}-3$ polyunsaturated fatty acids and the risk of rheumatoid arthritis: protocol for a cohort study using data from the Danish Diet, Cancer and Health cohort and Danish health registers. BMJ Open 2021;11:e047982. doi:10.1136/ bmjopen-2020-047982

- Prepublication history for this paper is available online. To view these files, please visit the journal online (http://dx.doi org/10.1136/bmjopen-2020047982).

Received 14 December 2020 Accepted 05 September 2021

Check for updates

(C) Author(s) (or their employer(s)) 2021. Re-use permitted under CC BY-NC. No commercial re-use. See rights and permissions. Published by BMJ.

For numbered affiliations see end of article.

Correspondence to Dr Bolette Gylden Soussi; b.soussi@rn.dk

\section{ABSTRACT}

Introduction Rheumatoid arthritis (RA) is a chronic autoimmune inflammatory joint disease with multifactorial aetiology. Smoking is a well-established lifestyle risk factor, but diet may also have an impact on the risk of RA. Intake of the major marine $n-3$ polyunsaturated fatty acids in particular eicosapentaenoic acid (EPA) and docosahexaenoic acid (DHA) have been hypothesised to lower the risk of RA due to their anti-inflammatory effects, although based on limited knowledge. Therefore, we aim to investigate the associations between dietary intake of EPA and DHA and the risk of incident RA.

Methods and analysis A cohort study. The follow-up design will be based on data from the Danish Diet, Cancer and Health cohort, which was established between 1993 and 1997. The participants will be followed through record linkage using nationwide registers including the Danish Civil Registration System, the Danish National Patient Registry and the Danish National Prescription Registry using the unique Civil Personal Registration number. Time-to-event analyses will be conducted with RA as the outcome of interest. The participants will be followed from inclusion until date of RA diagnosis, death, emigration or end of follow-up. HRs with $95 \%$ Cls obtained using Cox proportional hazard regression models, with age as underlying time scale and adjustment for established and potential risk factors, will be used as measures of association.

Ethics and dissemination The study has been approved by the Data Protection Committee of Northern Jutland, Denmark (2019-87) and the North Denmark Region Committee on Health Research Ethics (N-20190031). Study results will be disseminated through peer-reviewed journals and presentations at international conferences.

\section{INTRODUCTION}

Rheumatoid arthritis (RA) is the most common chronic autoimmune joint disease. $\mathrm{RA}$ is characterised by inflammation of the
Strengths and limitations of this study

Danish administrative registers ensure nearly complete follow-up of the study population.

- Use of a validated rheumatoid arthritis (RA) case definition with a positive predictive value of $88 \%$ for overall RA

- All exposures will be energy adjusted.

- Dietary assessment is based on a single food frequency questionnaire, which may not capture changes in dietary habits during the follow-up.

- Participants were 50-65 years old Caucasians at enrolment, and therefore, the results may not be valid to other age and ethnic groups.

synovial joints leading to irreversible joint damage and deformity, which may result in severe disability. The overall incidence rate of RA from 1996 to 2016 in Denmark has been reported to be 35 per 100000 person years. The incidence rate was approximately twofold higher in women than in men, and with a peak in incidence rate between ages of 70 and 74 in both sexes. ${ }^{1}$

The aetiology of RA is considered multifactorial and lifestyle factors seem to play an important role for the development of RA. Smoking is the best established lifestyle risk factor, ${ }^{2-5}$ whereas the impact of other lifestyle factors such as diet are less clear.

Marine n-3 polyunsaturated fatty acids (PUFAs) are organic compounds that may affect a variety of biological pathways which may in turn influence inflammatory processes. Marine n-3 PUFAs may, on ingestion, become incorporated into cellular membranes, pooled for storage or converted 
into lipid signalling molecules. The marine n-3 PUFAs, eicosapentaenoic acid (EPA) and docosahexaenoic acid (DHA), are mainly derived from seafood, especially fatty fish. ${ }^{6}$ PUFA seems to play an important role in the immune system. While the more common n-6 PUFAs mainly derived from vegetable oils and meat have proinflammatory actions, the marine n-3 PUFAs, EPA and DHA, has been ascribed anti-inflammatory properties by inhibiting a number of aspects of inflammation including production of cytokines and eicosanoids, leucocyte chemotaxis, adhesion molecule expression and leucocyte-endothelial adhesive interactions. ${ }^{78}$ Further, EPA and DHA promote production of inflammation resolving mediators in form of resolvins, protectins and maresins. ${ }^{8}$ Previous studies have supported that marine n-3 PUFAs may lower the risk of several inflammatory diseases including cardiovascular disease, inflammatory bowel disease and cancer. ${ }^{9}{ }^{10}$ However, limited data exist regarding the role of fish and marine n-3 PUFAs in relation to development of RA and findings have been conflicting. Thus, a previous cohort study, with a limited number of patients with RA, reported that intake of fatty fish was associated with a statistically non-significant lower risk of RA, whereas intake of medium fatty fish was associated with a significantly higher risk of RA compared with participants with a lower intake. ${ }^{11}$ A previous cohort study based on the Swedish Mammography Cohort reported that a intake of marine n-3 PUFAs (EPA, DHA and docosapentaenoic acid) of more than $0.21 \mathrm{~g}$ per day was associated with a markedly lower risk of RA compared with a lower intake. ${ }^{12}$ In contrast, a recent cohort study conducted among US women found no clear association between total marine n-3 PUFAs from diet and supplements and the risk of RA. ${ }^{13}$ Therefore, whether marine n-3 PUFAs may lower the risk of developing RA remains unclear.

The aim of this study is to assess the associations between dietary intake of EPA and DHA and the risk of incident RA. We hypothesise that intake of marine n-3 PUFAs, respectively, EPA and DHA is inversely associated with the rate of incident RA.

\section{METHODS AND ANALYSES \\ Study design}

We will conduct a cohort study using data from the Danish Diet, Cancer and Health (DCH) cohort and Danish health registers.

\section{Data sources}

We will follow the participants enrolled into the DCH cohort through nationwide Danish health registers including the Danish National Patient Registry (DNPR), the Danish National Prescription Registry (NPR) and the Civil Registration System (CRS). Use of the unique 10-digit personal identifier assigned to all Danish residents at birth or immigration, allows for accurate registerlinkage on an individual-based level.

\section{Diet, cancer and health}

The DCH cohort was established from December 1993 to May 1997 and includes participants from the area of greater Copenhagen and Aarhus in Denmark who did not have a previous cancer diagnosis registered in the Danish Cancer Registry prior to enrolment. In total, 57 053 subjects between 50 and 65 years of age were included in the study (27 179 men and 29874 women). At baseline, participants completed a detailed questionnaire on health status, social factors, lifestyle, and a validated 192item semiquantitative food frequency questionnaire. ${ }^{145}$ The food frequency questionnaire covered a total of 24 questions regarding intake of fish. The food frequency questionnaire has previously been validated against two times 7-day weighted diet records and found useful for categorising according to intake of total energy and PUFA intake. ${ }^{15}$ The questionnaires were checked for reading errors and missing information at baseline by a technician. Furthermore, anthropometric measurements were collected at baseline. A detailed description of the DCH cohort has been published previously. ${ }^{16}$

\section{Danish National Patient Registry}

The DNPR holds data on all admissions to somatic hospitals in Denmark since 1977 and all outpatient attendances since 1995. The data include dates of hospital admissions, ward types, discharge diagnoses, dates of all attendances at outpatient clinics and diagnoses recorded at each attendance. ${ }^{17}{ }^{18}$ Diseases are classified according to the International Classification of Disease (ICD). The ICD eighth edition was used until January 1994, and thereafter the ICD 10th edition was implemented.

\section{Danish National Prescription Registry}

The NPR provides data about all prescription drugs dispensed at Danish community pharmacies since $1994,{ }^{19}$ however, the register does not include information on drugs dispensed by hospital pharmacies directly to inpatients or outpatients.

\section{Civil Registration System}

The CRS is continuously updated and ensures complete follow-up regarding vital and migration status of all Danish citizens. ${ }^{20}$

\section{Study population}

The study population consists of all participants enrolled into the DCH cohort with complete records of data on exposures and covariates and without a diagnosis of RA recorded in the DNPR prior to their enrolment into the cohort. Also, participants with a previous diagnosis of cancer before enrolment will be excluded as well.

\section{Exposures and outcome of interest}

Intake of EPA and DHA will be calculated based on the food frequency questionnaire collected at baseline. By multiplying the frequencies of intake by the portion size, the individual average intake in grams per day of all foods and nutrients will be calculated using the software 
Table 1 ATC codes for conventional synthetic DMARD

\begin{tabular}{ll}
\hline Conventional synthetic DMARD & ATC code \\
\hline Methotrexate & L04AX03/L01BA01 \\
Sulfasalazine & A07EC01 \\
Hydroxychloroquine & P01BA02 \\
Leflunomide & L04AA13 \\
Azathioprine & L04AX01 \\
\hline
\end{tabular}

ATC, anatomical therapeutic chemical; DMARD, disease-modifying antirheumatic drug.

FoodCalc V.1.3 based on Danish Food composition tables used in Denmark during enrolment of the DCH participants. ${ }^{15} 21$ Exposures of interest include the energyadjusted intake of EPA and DHA.

The outcome of interest for this study is incident RA, defined as the first occurrence of an ICD-10 code of M0506, though not including M06.1, registered in the DNPR and a subsequent redeemed prescription of a conventional synthetic disease-modifying antirheumatic drug (csDMARD). Redeemed prescriptions of csDMARD are identified in the NPR by Anatomical Therapeutic Chemical (ATC) codes (table 1). A previous validation study by Linauskas et al reported in the same cohort a positive predictive value of $88 \%$ for overall RA based on this case definition. $^{22}$

Established risk factors for RA were identified by review of the existing literature prior to data analysis. We identified the following covariates that needed to be adjusted for: age (years), ${ }^{1}$ sex (women, men), ${ }^{123-25}$ smoking (smoking status (never, former or current smoker) and pack years of smoking (years)), ${ }^{3-5}$ level of education (basic school, higher education 1-2 year, higher education 3-4 year or higher education $>4$ years), ${ }^{3}$ alcohol (alcohol consumption (grams per day) and alcohol abstinence (yes, no) ), ${ }^{26}$ waist circumference $(\mathrm{cm}),{ }^{27}$ body fat percentage $(\%),{ }^{27}$ and physical activity (hours per week) ${ }^{28}$; and further for women: early menopause (yes, no), ${ }^{29}$ breast-feeding (months),${ }^{29}$ hormone replacement therapy $(<7, \geq 7$ years of use),${ }^{29}$ oral contraception $\left(<7, \geq 7\right.$ years of use),${ }^{29}$ and parity (number of pregnancies) ${ }^{29}$ Data on genetic disposition are not available and therefore not adjusted for.

\section{Statistical analysis plan}

Demographic and descriptive data will be presented as proportions (\%) for categorical variables and median values for continuous variables with $95 \%$ central ranges (2.5th; 97.5th percentile).

The participants will be followed from date of entry into the DCH cohort until date of RA diagnosis, death, emigration or end of follow-up (end of year 2018), whichever comes first.

We will perform time-to-event analyses with RA as the event of interest. HRs with 95\% CIs obtained from Cox proportional hazard regression models will be used as measures of association between dietary intake of EPA and DHA and rate of incident RA. The time-to-event analyses will be conducted by allowing for different baseline hazards among men and women with attained age as the underlying timescale, with adjustment for baseline age to ensure that baseline covariates have same age.

All exposure variables will be examined in continuous analyses using restricted cubic splines and in categorical analyses in quintiles. Radar plots will be constructed to support the interpretation of our results and to visualise potential confounding from the underlying dietary pattern.

A two-tailed $\mathrm{p}<0.05$ will be considered statistically significant.

Intake of EPA and DHA will be energy adjusted using the residual method assuming that intake of our exposures of interest are of most biological relevance relative to total energy intake. Residuals will be estimated separately among men and women based on linear regression between total energy intake and reported intakes of EPA and DHA. All estimated residuals will be added to the mean intake among men and women within the cohort. By definition, residual energy-adjusted intakes of EPA and DHA are independent of the total energy.

Based on the identified risk factors, three models were created (table 2). Model 1 contains adjustment for demographic risk factors. Model 2 (primary model for interpretation) contains the same covariates as model 1 combined with lifestyle risk factors. Differences in point estimates observed in analyses including adjustment for the covariates included in model 2 compared with model 1 may reflect the importance of confounding. In supplemented analyses, we will conduct sex-specific analyses. In analyses of the associations between EPA and DHA and the rate of RA in women additional adjustment for potential hormonal risk factors (model 3) will be undertaken to investigate residual confounding.

If we have few cases of RA it may be necessary to lower the number of risk factors that we adjust for.

In addition, secondary and sensitivity analyses will be conducted. In supplemental analyses, we will investigate our exposure of interest and the risk of seropositive and seronegative RA. Also, we will terminate the follow-up earlier to explore the influence of follow-up time on our associations of interest. Relevant sensitivity analyses include adjustment for risk factors using other available variables than the ones used in the primary analysis, for example, adjustment for body mass index (BMI) instead of body fat percentage. Also, we will adjust for duration (years) since smoking cessation in former smokers. Model 2 contains adjustment for education and we will add a variable for household income. In sensitivity analyses, we will examine whether the spline curves are robust when the number and location of the knots are modified. Further, our main analysis will be conducted with a less strict and a more restrict outcome definition, respectively.

The proportional hazards assumption will be evaluated by plotting the scaled Schoenfeld residuals against age. 
Table 2 Overview of models including adjustment for risk factors in each model

\begin{tabular}{llll}
\hline Covariates & Model 1 & Model 2* & Model 3† \\
\hline Demographic & Age & Age & Age \\
& Sex & Sex & Sex \\
Lifestyle & & Smoking & Smoking \\
& Education & Edcohol & Alcohol \\
& Waist circumference & Waist circumference \\
& Body fat percentage & Body fat percentage \\
Hormonal & Physical activity & Physical activity \\
& & Early menopause \\
& & Breast feeding \\
& & Hormone replacement therapy \\
\end{tabular}

*Model 2 will be considered the main analysis.

†Model 3 will be performed as a sex-specific supplemented analyses in women only.

The independent censoring assumption will be evaluated by adjustment for recruitment time.

\section{Patient and public involvement}

A patient research partner has been included in the development of the protocol and had the opportunity to submit comments to the protocol.

\section{DISCUSSION}

This study will explore the association between intake of the major marine n-3 PUFA, EPA and DHA, and the risk of incident RA. We will study a large number of cases, identified within a well-defined cohort (DCH) with nearly complete follow-up. Baseline age in the cohort was between 50 and 65 years and the sex and age distribution displays a slightly higher participation among women than among men. ${ }^{16}$

For each of the covariates selected for the models the following arguments apply. Age was an important risk factor for RA. RA can occur in patients of any age, but the peak onset was between the ages of 70 and 74 in both sexes. ${ }^{1}$ Sex was a known risk factor for RA, with an approximately twofold higher incidence in women compared with men. ${ }^{123-25}$ Current and previous smoking were associated with a higher risk of RA in both sexes, with both duration and intensity of importance. ${ }^{34}$ Thus, Liu et al conducted a cohort study among US women and showed that increased duration of smoking cessation was associated with a lower risk of overall RA in women, nevertheless, a higher risk of overall RA was still detectable after 30 years of smoking cessation compared with never smokers. ${ }^{5}$ With increasing levels of education the risk of RA decreased, which applied for both sexes. ${ }^{3}$ Low to moderate alcohol consumption, but not high alcohol consumption, was associated with a lower rate of incident
RA compared with no alcohol consumption. ${ }^{26}$ A higher body fat percentage, waist circumference and BMI were associated with higher risk of overall and other RA in women, whereas no clear association was found in men. ${ }^{27}$ For anthropometry measurements we selected the variables waist circumference and body fat percentage as a measurement of fat distribution. Leisure-time activity was associated with lower risk of incident RA in women. The lower risk of RA seemed to be dose-response related, as the findings were more pronounced in women who bicycled or walked more than 20 min per day and exercised more than 1 hour per week compared with those who did not. ${ }^{28}$ Because the highest incidence of RA occur in women after menopause several hormonal risk factors has been suggested. Early menopause is considered associated with higher risk of RA. ${ }^{29}$ In contrast, breast feeding, use of hormone replacement therapy and oral contraception over 7 years has been mentioned as protective factors for RA. ${ }^{29}$ The association between parity and risk of RA is less clear, and parity has been suggested both to be a risk and a protective factor. ${ }^{29}$

This study will not likely be prone to systematic problems with misclassification on exposure level and selection bias, since all exposure variables and covariates were collected before and independently of the outcome of interest. However, it has previously been shown that the case definition of RA affects the estimates of its incidence and prevalence. ${ }^{24}$ Therefore, a strength of this study is that cases will be identified using a validated case definition of RA that rely on linkage between Danish health registers. Furthermore, we will conduct sensitivity analyses based on a less and more restrictive case definition of RA, respectively.

Measurements of both our exposure (EPA and DHA) and outcome (incident RA) variables are believed to be of 
high quality, ${ }^{152}$ and the intake of n-3 PUFA in the DCH cohort were higher than in other western populationbased studies. However, the information on dietary intake of EPA and DHA will be based on a single food frequency questionnaire and repeated dietary measurements would have been preferable in order to capture changes in dietary habits over time and to reduce random measurement errors. We believe the intake of EPA and DHA are of most biological relevance relative to the total energy intake, and therefore, we will use the residual method to energy-adjust all exposures. Adjustment for total energy is obtained by this method. By applying an analytical method as energy adjustment the risk of measurement error in dietary exposures is reduced. ${ }^{30}$

Potential confounders identified prior to data analysis are included in our statistical models. The underlying dietary pattern will be explored using radar plots to identify any potential confounding from the underlying dietary pattern in the study population. Based on the design and available data, potential residual confounding can occur due to inaccurate self-reported information from the DCH questionnaires, insufficient adjustment or risk factors not identified and incorporated into the statistical model.

Patients with RA from private practising rheumatologists in primary care will not be included in our study, unless they have been transferred to a hospital-based rheumatologist or admitted to a hospital for any reason. Therefore, our findings might not be generalised to mild RA cases treated out of hospital. The DCH data were obtained from the area of greater Copenhagen and Aarhus and finding may therefore not be generalised to the entire Danish population. Of the invited subjects in the DCH cohort study, only $35 \%$ accepted to participate in the study. ${ }^{16}$ A previous study has indicated that participants within the DCH cohort was socioeconomically more affluent than non-participants, suggesting that our study sample may be healthier than the general population. ${ }^{16}$ Moreover, all participants were Caucasians and at least 50 years of age at inclusion, and therefore, may our findings not be valid in younger age or other ethnicity groups.

This study is expected to bring new insight into the association between dietary intake of marine n-3 PUFAs and the risk of development of RA. The study may potentially bring new insights into the pathogenesis of RA, and it may redefine our understanding of how dietary intake of marine n-3 PUFA, EPA and DHA, may influence the risk of RA.

\section{Ethics and dissemination}

The study has been approved by the North Denmark Region Committee on Health Research Ethics (N-20190031) and the Data Protection Committee of Northern Jutland, Denmark (2019-87). The DCH cohort was approved by the relevant scientific Ethic Committees and the Data Protection Agency and all participants gave written informed consent at enrolment. The results of this study will be published in an international peer-reviewed journal and disseminated at international conferences.

Author affiliations

${ }^{1}$ Department of Rheumatology, Aalborg University Hospital, Aalborg, Denmark ${ }^{2}$ Department of Cardiology, Aalborg University Hospital, Aalborg, Denmark ${ }^{3}$ Department of Clinical Medicine, Aalborg University, Aalborg, Denmark ${ }^{4}$ Unit of Clinical Biostatistics, Aalborg University Hospital, Aalborg, Denmark ${ }^{5}$ Aalborg AF Study Group, Aalborg University Hospital, Aalborg, Denmark ${ }^{6}$ Department of Nephrology, Aalborg University Hospital, Aalborg, Denmark

Twitter Bolette Gylden Soussi @BoletteSoussi

Acknowledgements The authors will like to thank the patient research partner for the contribution to the protocol.

Contributors The study idea was established by BGS, LD, SK, EBS and JHC and the protocol was finalised in collaboration with CSB. All authors (BGS, CSB, SK, SLC, KD, RLC, JHC, EBS and LD) contributed to methodological considerations. BGS, CSB, SL-C, KD and LD were involved in the statistical analysis plan. The first draft of the manuscript was written by BGS. All authors (BGS, CSB, SK, SL-C, KD, RLC, JHC, EBS and LD) contributed to the final manuscript, critically reviewed the manuscript and agreed on submission.

Funding This work was supported by the Danish Rheumatism Association (R172A6090; R175-A6091; R186- A6573, Bolette Gylden Soussi) and Aase and Ejnar Danielsens Foundation (19-10-0295, Bolette Gylden Soussi).

Competing interests BGS reports grants from The Danish Rheumatism Association, grants from Aase and Ejnar Danielsens Foundation, during the conduct of the study. CSB has nothing to disclose. SK has nothing to disclose. SL-C has nothing to disclose. KD has nothing to disclose. RLC has nothing to disclose. JHC has nothing to disclose. EBS has nothing to disclose. LD reports grants from BMS, other from Galderma, other from Eli Lilly, outside the submitted work.

Patient and public involvement Patients and/or the public were involved in the design, or conduct, or reporting, or dissemination plans of this research. Refer to the Methods section for further details.

Patient consent for publication Not applicable.

Provenance and peer review Not commissioned; externally peer reviewed.

Open access This is an open access article distributed in accordance with the Creative Commons Attribution Non Commercial (CC BY-NC 4.0) license, which permits others to distribute, remix, adapt, build upon this work non-commercially, and license their derivative works on different terms, provided the original work is properly cited, appropriate credit is given, any changes made indicated, and the use is non-commercial. See: http://creativecommons.org/licenses/by-nc/4.0/.

ORCID iDs

Bolette Gylden Soussi http://orcid.org/0000-0001-5759-7942

Christian Sørensen Bork http://orcid.org/0000-0001-5233-2902

\section{REFERENCES}

1 Soussi BG, Cordtz RL, Kristensen S. OP0068 incidence and prevalence of rheumatoid arthritis in denmark: a nationwide population-based study investigating the effect of four different case definitions. Ann Rheum Dis 2020;79:46.

2 Liao KP, Alfredsson L, Karlson EW. Environmental influences on risk for rheumatoid arthritis. Curr Opin Rheumatol 2009;21:279-83.

3 Reckner Olsson A, Skogh T, Wingren G. Comorbidity and lifestyle, reproductive factors, and environmental exposures associated with rheumatoid arthritis. Ann Rheum Dis 2001;60:934-9.

4 Criswell LA, Merlino LA, Cerhan JR, et al. Cigarette smoking and the risk of rheumatoid arthritis among postmenopausal women: results from the lowa women's health study. Am J Med 2002;112:465-71.

5 Liu X, Tedeschi SK, Barbhaiya M, et al. Impact and timing of smoking cessation on reducing risk of rheumatoid arthritis among women in the nurses' health studies. Arthritis Care Res 2019;71:914-24.

6 Rimm EB, Appel LJ, Chiuve SE, et al. Seafood long-chain n-3 polyunsaturated fatty acids and cardiovascular disease: a science Advisory from the American heart association. Circulation 2018;138:35-47.

7 Navarini L, Afeltra A, Gallo Afflitto G, et al. Polyunsaturated fatty acids: any role in rheumatoid arthritis? Lipids Health Dis 2017;16:197. 
8 Calder PC. Marine omega-3 fatty acids and inflammatory processes: effects, mechanisms and clinical relevance. Biochim Biophys Acta 2015;1851:469-84.

9 Yates CM, Calder PC, Ed Rainger G. Pharmacology and therapeutics of omega-3 polyunsaturated fatty acids in chronic inflammatory disease. Pharmacol Ther 2014;141:272-82.

10 Simopoulos AP. Omega-3 fatty acids in health and disease and in growth and development. Am J Clin Nutr 1991;54:438-63.

11 Pedersen M, Stripp C, Klarlund M, et al. Diet and risk of rheumatoid arthritis in a prospective cohort. J Rheumatol 2005;32:1249-52.

12 Di Giuseppe D, Wallin A, Bottai M, et al. Long-term intake of dietary long-chain n-3 polyunsaturated fatty acids and risk of rheumatoid arthritis: a prospective cohort study of women. Ann Rheum Dis 2014;73:1949-53.

13 Sparks JA, O'Reilly Éilis J, Barbhaiya M, et al. Association of fish intake and smoking with risk of rheumatoid arthritis and age of onset: a prospective cohort study. BMC Musculoskelet Disord 2019;20:2.

14 Overvad K, Tjønneland A, Haraldsdóttir J, et al. Development of a semiquantitative food frequency questionnaire to assess food, energy and nutrient intake in Denmark. Int $J$ Epidemiol 1991;20:900-5

15 Tjønneland A, Overvad K, Haraldsdóttir J, et al. Validation of a semiquantitative food frequency questionnaire developed in Denmark. Int J Epidemiol 1991;20:906-12.

16 Tjønneland A, Olsen A, Boll K, et al. Study design, exposure variables, and socioeconomic determinants of participation in diet, cancer and health: a population-based prospective cohort study of 57,053 men and women in Denmark. Scand J Public Health 2007;35:432-41.

17 Lynge E, Sandegaard JL, Rebolj M. The Danish national patient register. Scand J Public Health 2011;39:30-3.

18 Schmidt M, Schmidt SAJ, Sandegaard JL, et al. The Danish nationa patient registry: a review of content, data quality, and research potential. Clin Epidemiol 2015;7:449-90.
19 Pottegård A, Schmidt SAJ, Wallach-Kildemoes H, et al. Data resource profile: the Danish national prescription registry. Int $J$ Epidemiol 2017;46:798-798f.

20 Schmidt M, Pedersen L, Sørensen HT. The Danish civil registration system as a tool in epidemiology. Eur J Epidemiol 2014;29:541-9.

21 Lauritsen J. Foodcalc V. 1.3, 1998. Available: https://www.cancer.dk/ dyn/resources/File/file/7/8207/1570007155/foodcalc_documentation. pdf [Accessed 02 Nov 2020].

22 Linauskas A, Overvad K, Johansen MB, et al. Positive predictive value of first-time rheumatoid arthritis diagnoses and their serological subtypes in the Danish national patient registry. Clin Epidemiol 2018;10:1709-20.

23 Pedersen JK, Svendsen AJ, Hørslev-Petersen K. Incidence of rheumatoid arthritis in the southern part of Denmark from 1995 to 2001. Open Rheumatol J 2007;1:18-23.

24 Eriksson JK, Neovius M, Ernestam S, et al. Incidence of rheumatoid arthritis in Sweden: a nationwide population-based assessment of incidence, its determinants, and treatment penetration. Arthritis Care Res 2013;65:870-8.

25 Myasoedova E, Davis J, Matteson EL, et al. Is the epidemiology of rheumatoid arthritis changing? results from a population-based incidence study, 1985-2014. Ann Rheum Dis 2020;79:440-4.

26 Jin Z, Xiang C, Cai Q, et al. Alcohol consumption as a preventive factor for developing rheumatoid arthritis: a dose-response metaanalysis of prospective studies. Ann Rheum Dis 2014;73:1962-7.

27 Linauskas A, Overvad K, Symmons D, et al. Body fat percentage, waist circumference, and obesity as risk factors for rheumatoid arthritis: a Danish cohort study. Arthritis Care Res 2019;71:777-86.

28 Di Giuseppe D, Bottai M, Askling J, et al. Physical activity and risk of rheumatoid arthritis in women: a population-based prospective study. Arthritis Res Ther 2015;17:40.

29 Deane KD, Demoruelle MK, Kelmenson LB, et al. Genetic and environmental risk factors for rheumatoid arthritis. Best Pract Res Clin Rheumatol 2017;31:3-18.

30 Willett W, Stampfer MJ. Total energy intake: implications for epidemiologic analyses. Am J Epidemiol 1986;124:17-27. 